2. Blocks are dehydrated in ethyl alcohol in the usual way.

3. Blocks are immersed in a $3 \%$ solution of low viscosity nitrocellulose* in methyl benzoate for two to three hours, then transferred to a fresh $3 \%$ solution for a further two to three hours, and afterwards to a $20 \%$ solution for a further 18 hours.

4. After carefully removing the excess low viscosity nitrocellulose the block is immersed for two hours in each of three changes of benzene.

5. The block is placed in wax (melting point $56^{\circ} \mathrm{C}$.) overnight and then blocked out in fresh wax in the usual way.

\section{Section Cutting and Mounting} calcified from non-calcified bone. Sections of undecalcified cancellous bone enable this distinction to be made without the uncertainties that may attend the interpretation of stained decalcified tissue. Undecalcified sections suitable for microscopy have been used by several workers investigating bone growth in young animals (McLean and Bloom, 1940; Duthie, 1954), but they are apparently rarely employed in routine histopathological practice.

In this laboratory, during the last few years, all biopsies from suspected cases of metabolic bone disease have been routinely reported from undecalcified sections; and our experience has shown that these can be prepared without special skill or intricate apparatus. Sections from blocks decalcified in aqueous solutions of the tetra-sodium salt of ethylene-diamine-tetra-acetic acid (adjusted to $p \mathrm{H} \mathrm{7.0-7.2)} \mathrm{have} \mathrm{also} \mathrm{been} \mathrm{routinely}$ prepared. These have not proved as reliable as undecalcified sections in distinguishing between calcified bone and osteoid (and usually take more time to prepare), but they are helpful when used alongside undecalcified sections for studying bone structure and surveying the general topography of the bone lesions.

No originality is claimed for the method of preparing undecalcified sections which is presented below, as it introduces no new technical principles; but its simplicity may encourage others to try it, and the ease and certainty with which osteoid can be recognized are a great help in histological diagnosis.

\section{Source of Material}

It is advisable to choose an area for biopsy distant from local lesions, such as Looser's zones, or fractures. A coring, or preferably a wedge, from the iliac crest is the usual source of material. Small $(5 \mathrm{~mm}$.) blocks of cancellous bone are cut out of the specimen.

\section{Preparation of Blocks}

1. Blocks are fixed overnight in $5 \%$ formalin $(2 \%$ formaldehyde) in veronal $\mathrm{HCl}$ buffer at $p \mathrm{H}$ 7.2. Specimens which have been immersed in "neutral " formolsaline for a short period are acceptable for diagnostic purposes.
The block is cut on an ordinary sledge microtome, the small knives used on rocking microtomes being insufficiently robust. The central part of a length of plastic adhesive tape (e.g., "speedfix") is pressed on to the surface of the block and, holding the forward end of the tape free of the knife edge, a section is taken at 7-8 $\mu$. The tape is then re-applied to the block and a further section taken. The tape is invaluable; it prevents impaction and curling of the sections which may be cut serially even by junior technicians.

A part of the tape with one or more sections fixed to it is then pressed, adhesive face downwards, on to an albuminized slide. The plastic backing and the adhesive on the tape are then removed by immersing the slide overnight in xylol or a similar solvent, depending on the properties of the particular tape used.

Sections are carried through alcohol to distilled water. When mounted in distilled water, calcified and noncalcified bone can usually be distinguished by optical differences with ordinary illumination or with polarized light; if such a section is stained in daylight with a filtered $1 \%$ aqueous solution of silver nitrate for 10 minutes and then thoroughly rinsed in distilled water, the stained areas correspond very closely with the calcified bone seen in the unstained specimen (Fig. 1). routinely counterstained with $0.1 \%$ safranin buffered to $p \mathrm{H} \mathrm{7.2,} \mathrm{then} \mathrm{dehydrated} \mathrm{in} \mathrm{alcohol} \mathrm{and} \mathrm{mounted} \mathrm{in}$ DPX; Canada balsam is better avoided. Generally the soft tissue structure and the cellular staining properties are excellently preserved.

More colourful effects can be obtained by staining either the untreated or silver-stained section with haemalum and eosin. If used without prior silver staining some care is required as aqueous solutions of haemalum are sufficiently acid to decalcify the section in time; but if the staining with Mayer's haemalum is limited to one minute no serious loss of bone salt occurs and the results are essentially the same as in silver-stained sections. The dificulty with acid dye solutions is easily demonstrated by observing the formation and

* Obtainable from Messrs. George T. Gurr, London. However, in this laboratory the silver-stained section is 

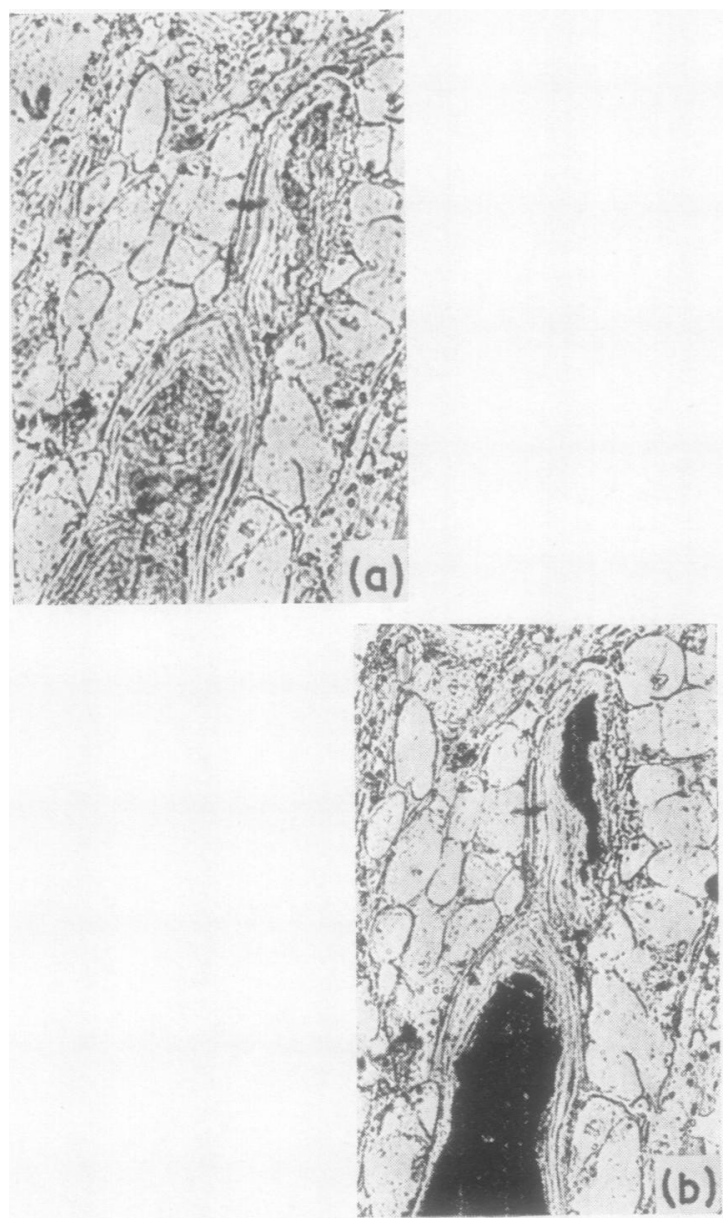

FIG. 1-Undecalcified section mounted in water: (a) unstaiıled (b) stained with silver nitrate. The black calcified bone in (b) closely corresponds with the calcified area in (a). The soft tissue structure is preserved; in both (a) and (b) the osteoid seam surrounding the calcified trabecula is obvious. $\times 120$.

disappearance of the red calcium lake formed in sections covered with aqueous alizarin $(p \mathrm{H} \mathrm{2.5).} \mathrm{Alizarin}$ buffered to neutrality has not proved a useful calcium stain. Most information is obtained by staining some sections by the silver-safranin method and others by haemalum and eosin with or without previous silver staining.

\section{REFERENCES}

Duthie, R. B. (1954). J. Path. Bact., 68, 296.

McLean, F. C., and Bloom, W. (1940). Anat. Rec., 78, 333.

\section{A Rapid Method for Estimating Fibrinogen in Plasma}

\author{
M. COLES AND W. ROMAN \\ From the Biochemistry Division, Institute of Medical and \\ Veterinary Science, Adelaide, South Australia
}

(RECEIVED FOR PUBLICATION OCTOBER 18, 1956)

The letter by Dr. G. L. Robinson in the British Medical Journal (1956) encourages us to publish our experience with the turbidimetric method of Parfentjev, Johnson, and Cliffton (1953) which Fowell (1955) elaborated, but which does not seem to be known in either the U.K. or Australia.

With the availability of fibrinogen solutions for intravenous administration, the rapid estimation of fibrinogen in plasma, especially in post-partum haemorrhage or conditions associated with fibrinogenopenia, becomes of ever-increasing importance as has also been shown by FitzGerald and Jackson (1956). The classical method, still used by FitzGerald and Jackson, is too cumbersome and takes too long in emergencies and none of the so. called bedside tests are wholly satisfactory, as Dr. Robinson points out in his letter. The turbidimetric method can be carried out by any technician who can use a colorimeter in 10 to 15 minutes and requires only a simple colorimeter which is available these days in all hospital laboratories. We use either the "unicam" SP 300 with the blue-green filter or SP 400 at a wavelength of $500 \mathrm{~m} \mu$.

Parfentjev et al. found that only fibrinogen is precipitated from plasma with $20 \%$ saturated ammonium sulphate solution and that 1 part of plasma or diluted plasma with 9 parts of an ammonium sulphate solution containing $133 \mathrm{~g}$. litre will give a final ammonium sulphate concentration of $12 \%$ which causes a turbidity proportionate to the fibrinogen concentration. We found that this turbidity obeys Beer's law over the range 0.05 $1.0 \%$ and direct proportionality between optical density and fibrinogen concentration is obtained after deduction of a blank value due to the natural turbidity of the plasma under test when using the above-mentioned wavelengths and colorimeters.

\section{Method}

Citrated or oxalated blood is used and centrifuged at 2,000 r.p.m. for five minutes, the plasma taken off and recentrifuged under the same conditions. Two test tubes are set up, the blank containing $0.5 \mathrm{ml}$. of plasma and $9.5 \mathrm{ml}$. normal saline, the test $0.5 \mathrm{ml}$. plasma, $0.5 \mathrm{ml}$. normal saline, and $9 \mathrm{ml}$. ammonium sulphate reagent. Eoth tukes are mixed by gentle inversion (no shaking, since this may lead to partial defibrination and uneven turbidity). Exactly three minutes (timed with a stopwatch) after the addition of the ammonium sulphate to the plasma under test, its optical density is read in the 